\title{
Determining nuclear shape
}

\section{The role of farnesylated nuclear membrane proteins}

\begin{abstract}
Maria Polychronidou ${ }^{1,2}$ and Jörg Großhans*
${ }^{1}$ Institut für Biochemie und Molekulare Zellbiologie; Universitätsmedizin; Georg-August-Universität Göttingen; Göttingen, Germany;

${ }^{2}$ Zentrum für Molekulare Biologie der Universität Heidelberg (ZMBH); ZMBH-DKFZ Allianz; Heidelberg, Germany
\end{abstract}

Key words: nuclear shape, lamin, kugelkern, nuclear membrane, farnesylation, lipid bilayer

Submitted: $10 / 12 / 10$

Revised: $10 / 19 / 10$

Accepted: 10/20/10

DOI: 10.4161/nucl.2.1.13992

*Correspondence to: Jörg Großhans;

Email: joerg.grosshans@medizin.uni-goettingen.de
$\mathrm{C}$ hanges in nuclear morphology are observed in diverse developmental processes as well as in pathological conditions. Modification of nuclear membrane and nuclear lamina protein levels results in altered nuclear shapes, as it has been demonstrated in experimental systems ranging from yeast to human cells. The important role of nuclear membrane components in regulating nuclear morphology is additionally highlighted by the abnormally shaped nuclei observed in diseases where nuclear lamina proteins are mutated. Even though the effect of nuclear envelope components on nuclear shape has been thoroughly described, not much is known about the molecular mechanisms that govern these events. In addition to the known role of intermediate filament formation by lamins, here we discuss several mechanisms that might alone or in combination participate in the regulation of nuclear shape observed upon modification of the levels of nuclear membrane and lamina proteins. Based on recent work with the two farnesylated nuclear membrane Drosophila proteins, kugelkern and lamin Dm0, we propose that the direct interaction of farnesylated nuclear membrane proteins with the phospholipid bilayer leads to nuclear envelope deformation. In addition to this mechanism, we suggest that the interaction of nuclear membrane and lamina proteins with cytoskeletal elements and chromatin, and modifications in lipid biosynthesis might also be involved in the formation of abnormally shaped nuclei.

\section{The Nuclear Lamina}

The nuclear envelope (NE) is composed of an outer nuclear membrane (ONM) and an inner nuclear membrane (INM). The ONM is continuous with the endoplasmic reticulum (ER). The INM faces the interior of the nucleus and is characterized by the presence of a number of integral membrane proteins. In metazoans, a meshwork of proteins called the nuclear lamina underlies the INM. The lamina attaches to the INM via INM proteins and it also interacts with parts of the chromatin, either directly or via chromatin binding proteins. ${ }^{1,2}$ One of the numerous functions of the nuclear lamina is maintaining the mechanical properties of the nucleus and of the whole cell, by interacting with the cytoskeleton. ${ }^{3}$

The lamina is mainly composed of lamins and lamin interacting proteins. No lamins have been found in yeast ${ }^{4}$ and it remains unclear whether a structural or functional equivalent to the nuclear lamina exists in the yeast nucleus. ${ }^{5}$ Lamins are nuclear-specific Type $\mathrm{V}$ intermediate filaments, classified in A- and B-type. They share a common polypeptide structure, consisting of a short N-terminal head domain followed by an $\alpha$-helical rod domain and a globular C-terminal tail domain. The C-terminal residues match the CaaX farnesylation motif. B-type lamins are permanently farnesylated while the C-terminal motif of lamin A is proteolytically processed, leading to loss of the farnesylated residue. ${ }^{6}$ Mutations resulting in defective lamin or lamin binding proteins are associated to 
a variety of human diseases, collectively called laminopathies. ${ }^{7-9}$ Laminopathies include neuromuscular disorders (EmeryDreifuss muscular dystrophy, CharcotMarie-Tooth disease and dilated cardiomyopathy), lipodystrophies and progeria syndromes [Hutchinson-Gilford progeria syndrome (HGPS) and atypical Werner syndrome].

\section{Nuclear Shape Changes in Development and Disease: The Role of Nuclear Membrane and Lamina Components}

The nuclei of most eukaryotic cells are generally round or oval shaped and smooth. Nevertheless, alterations in nuclear morphology are observed in various physiological processes and pathologies. A classical example of nuclear shape changes under physiological conditions are the nuclei of neutrophil granulocytes..$^{10,11}$ During cell differentiation, the granulocyte nucleus adopts a lobulated shape, assumed to facilitate cell function, which involves migration through narrow tissue openings. ${ }^{12}$ Other examples of dramatically altered nuclear morphology in differentiating cells are the nuclei of spermatocytes $^{13}$ and of myocytes. ${ }^{14}$ Developmental nuclear shape changes are also observed in the early Drosophila embryo, where the shape of the cortical nuclei changes from spherical to ellipsoidal, showing a 2.5-fold increase in length. ${ }^{15-17}$ In addition to their increased length the nuclei show apical ruffling of the NM. Nuclear shape changes are associated with physiological organismal aging of $C$. elegans, D. melanogaster and humans. ${ }^{18-20}$ Among the pathologies where nuclear shape changes are observed are cancer ${ }^{21}$ as well as laminopathies. ${ }^{8}$

Generally, the molecular mechanisms that participate in defining nuclear shape in the above mentioned situations have remained unclear. ${ }^{22}$ As indicated by the abnormal nuclear shapes observed in diseases caused by mutations in NM components and lamina proteins, the NM seems to play an active role in determining nuclear shape. ${ }^{7}$ A characteristic example is the Pelger-Hüet anomaly (PHA) caused by mutations in the gene encoding the lamin $B$ receptor (LBR). ${ }^{23}$ The neutrophil granulocyte nuclei of PHA patients show hypolobulation and altered chromatin organization, indicating that the LBR participates in shaping the granulocyte nucleus.

Both depletion and overexpression of lamins result in aberrant nuclear shapes. Filament formation mediated by the rod domain of lamins is undoubtedly important for the definition of nuclear shape and the maintenance of nuclear integrity. ${ }^{24,25}$ Nevertheless, upon overexpression of lamins, their farnesylated C-terminal part seems to play a prominent role in the induction of nuclear abnormalities. A striking example is HGPS, a laminopathy caused by a point mutation in exon 11 of $L M N A$, activating a cryptic splice site and resulting in the formation of a permanently farnesylated variant of lamin A, La $\Delta 50 /$ progerin. ${ }^{26,27}$ Accumulation of $\mathrm{La} \Delta 50$ /progerin results in the formation of abnormally shaped nuclei with NM blebs and infoldings. ${ }^{28}$ The activity of $\mathrm{La} \Delta 50 /$ progerin on nuclear shape seems to be dependent on farnesylation, since the use of farnesyltransferase inhibitors (FTIs) restores the nuclear morphology defects. ${ }^{29,30}$ The farnesylated C-terminal part of lamins was also shown to be important for modulating nuclear shape by studies where lamin variants consisting only of the C-terminal part were tested for their activity. ${ }^{31,32}$ In these studies it was shown that farnesylated truncated lamin constructs lacking the filament forming domain are able to induce formation of abnormally shaped nuclei, in contrast to non-farnesylatable mutants or the non-farnesylatable lamin $\mathrm{C}$, which have no activity on nuclear morphology. Importantly, a lamin $\mathrm{C}$ mutant containing the CaaX of lamin Dm0, was able to induce NM abnormalities indicating that the CaaX motif is directly implicated in the nuclear morphology changes. ${ }^{32}$

Apart from lamins, the only farnesylated NM protein described so far is the Drosophila INM protein kugelkern (Kuk). ${ }^{33}$ The polypeptide structure of Kuk is somewhat similar to the one of lamins, containing a putative N-terminal coiledcoil motif and a C-terminal CaaX motif. Apart from the structural similarities, Kuk shares functional similarities with lamins, since Kuk overexpression results in aberrant nuclear shapes in different cell types (Fig. 1A-C). ${ }^{18,33,34}$ Furthermore, overexpression of Kuk in the adult fly results in shorter lifespan and aging related phenotypes similarly as when $\mathrm{La} \Delta 50$ / progerin is expressed in mice or humans. ${ }^{18}$ Reduced Kuk levels also affect nuclear morphology. In kuk deficient cellularizing Drosophila embryos, the nuclei fail to elongate and do not show apical NM ruffling. ${ }^{33}$ Furthermore, the nuclei seem to be detached from the centrosomes ${ }^{35}$ which might indicate that Kuk mediates the connection of NM to cytoskeletal elements. The activity of Kuk on nuclear elongation seems to be specific, since the kuk mutant phenotype cannot be rescued by overexpression of lamin $\mathrm{Dm} 0$. $^{34}$

Another example of a nuclear protein influencing nuclear shape is Esclp, a yeast membrane-associated component of the nuclear periphery ${ }^{36}$ that mediates the interaction of chromatin with the NE. Despite its coiled coil motifs Esclp does not seem to form filaments and it is generally not comparable to lamins. When Esclp is overexpressed it induces formation of lobulated and abnormally shaped interphase nuclei. ${ }^{5}$ The solubilization properties of Esclp suggest that it bears a not yet defined lipid modification, ${ }^{36}$ indicating that it could represent another example of a lipid modified protein that affects nuclear shape.

\section{Farnesylated Nuclear Membrane Proteins Affect Nuclear Shape by Directly Interacting with the Nuclear Membrane via their Lipophilic C-Terminus}

Triggered by the observation that the C-terminal lipophilic part of farnesylated NM proteins seems to be sufficient for their effect on nuclear shape we investigated how the C-terminal part affects the NM. In our recent study ${ }^{34}$ we focused on the two farnesylated Drosophila NM proteins, lamin Dm0 and Kuk. Strikingly, we observed that expression of lamin Dm0 and Kuk in yeast, results in the formation of enlarged nuclei, bearing NM lobes and protrusions (Fig. 1C). The modified nuclear shapes observed upon expression of lamin $\mathrm{Dm} 0$ and Kuk in yeast where 
there is no classical nuclear lamina, indicate that the existence of a lamina is not required for the activity of farnesylated proteins. Furthermore, LaminDm $0 \Delta \mathrm{N}$, a truncated lamin $\mathrm{Dm} 0$ construct lacking the entire N-terminal filament forming part showed activity on nuclear shape in mouse fibroblasts and yeast. This observation implies that intermediate filament formation does not seem to be essential for affecting nuclear shape.

Considering the indications for a lamina and filament independent mechanism, we tested the activity of recombinant lamin $\mathrm{Dm} 0$ and Kuk protein constructs on protein free liposomes. We used full length Kuk, two truncated Kuk constructs lacking parts of the $\mathrm{N}$-terminal half of the protein, LaminDm $0 \Delta \mathrm{N}$, and two GFP-fusion constructs and liposomes consisting mainly of negatively charged phosphatidylinositol and phosphatidylserine. GFP-NLS-Cterm consists of GFP fused to the NLS of lamin Dm0 followed by the C-terminus of lamin Dm0 without the globular part whereas GFP-NLSCaaX consists of GFP fused to the NLS and the CaaX of lamin Dm0. Consistent with the weak partition properties of farnesyl residues, we observed that binding to liposomes is not complete. We also observed that binding of the constructs to the liposomes is not absolutely dependent on farnesylation. This indicates that other parts of the protein and additional factors may participate in the interaction of farnesylated proteins with the NM. Our results come in agreement with previous studies showing that farnesylation is necessary, but not sufficient for targeting lamins to the NM. ${ }^{37-39}$ Importantly, when we examined the morphology of the liposomes by fluorescence microscopy we found that the proteins deform the liposomes in a farnesylation dependent way, ${ }^{34}$ meaning that binding to the liposomes was accompanied by morphological changes only when the proteins were farnesylated. In addition, we observed that the C-terminal globular domain of lamin $\mathrm{Dm} 0$ seems to contribute to the activity of the protein in membrane deformation, since GFPNLS-Cterm and GFP-NLS-CaaX showed activity on liposome morphology only at ten times higher concentration than Lamin $\mathrm{Dm} 0 \Delta \mathrm{N}$, despite binding to the
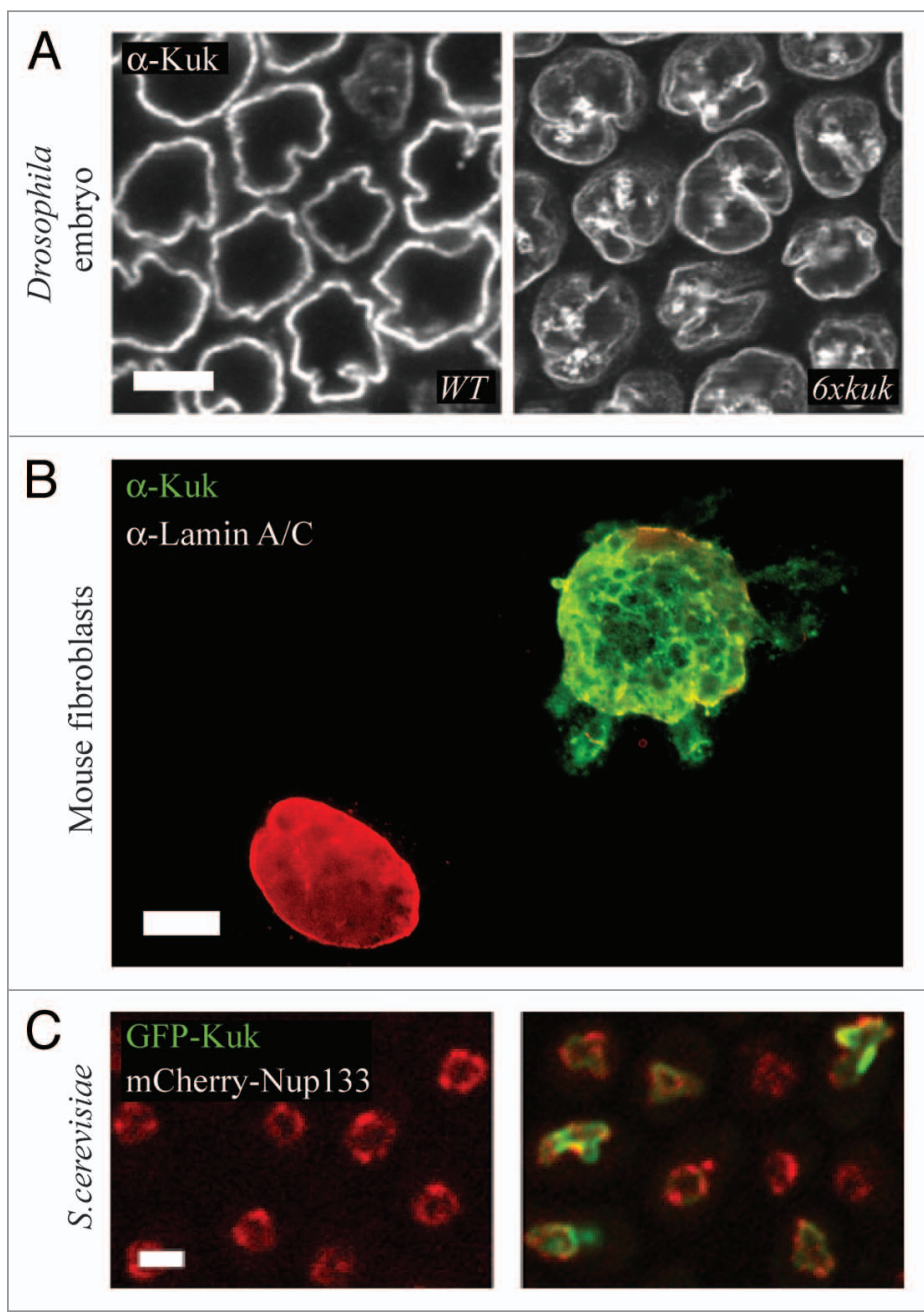

Figure 1. The farnesylated nuclear membrane protein Kuk affects nuclear shape in different cell types. (A) Surface view of a wt Drosophila embryo (left) and of an embryo with six genomic copies of kuk (right), in late cellularization. Kuk is used as a marker of the NM. Scale bar: $10 \mu \mathrm{m}$. (B) Nuclear morphology upon Kuk overexpression in NIH-3T3 cells. The nucleus of a transiently transfected $\mathrm{NIH}-3 \mathrm{~T} 3$ cell expressing Kuk (green) is shown together with the nucleus of a non transfected cell. Lamin A/C (red) marks the NM. Scale bar: $5 \mu \mathrm{m}$. (C) Nuclear morphology of control (left) and GFP-Kuk expressing (right) S. cerevisiae in mid-log phase. mCherry-Nup133 (red) marks the NM and GFP-Kuk is shown in green. Scale bar: $2.5 \mu \mathrm{m}$.

liposomes. The reduced activity of the two GFP-fusion constructs was also observed in transfected mouse fibroblasts.

Taking into consideration the observations discussed above we propose that the abnormalities in nuclear shape observed upon overexpression of farnesylated NM proteins might be directly arising from the insertion of the proteins to the lipid bilayer of the NM via their lipophilic farnesylated moiety. Since we observed that deletion of the C-terminal globular part of lamin Dm0 noticeably reduces the activity of the protein on the NM and on liposomes, we suggest that the globular part plays an important role in affecting nuclear shape. Kuk does not seem to contain a homologous globular domain, but we cannot exclude the possibility that structured domains in its C-terminus confer membrane deforming activity. In our current model shown in Figure 2, we propose that the association of farnesylated NM proteins with the lipid bilayer of the NM via their modified CaaX motif, results in the positioning of C-terminal protein domains in close contact with the NM, thus mediating structural changes in the lipid bilayer and finally inducing abnormal NM shapes. 


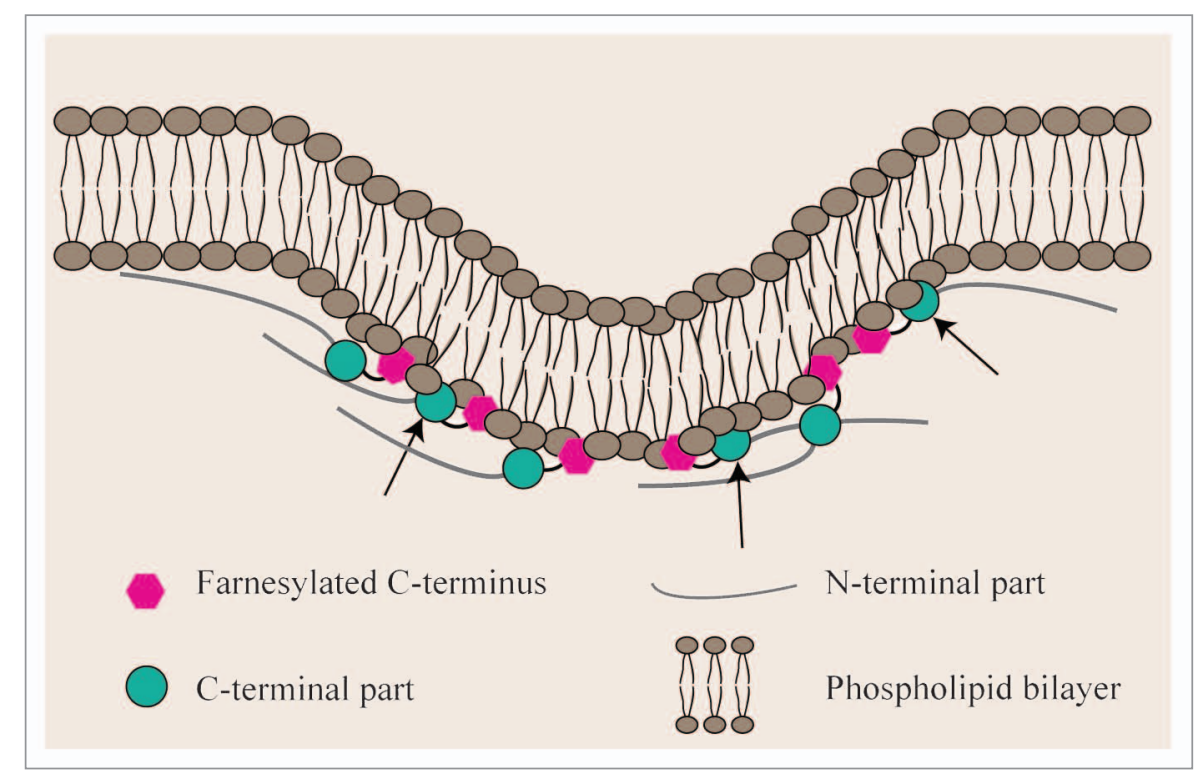

Figure 2. Farnesylated nuclear membrane proteins affect nuclear shape by directly interacting with the lipid bilayer. Schematic representation of our current model, explaining how farnesylated proteins of the nuclear membrane affect nuclear shape by directly interacting with the lipid bilayer of the INM via their farnesylated C-terminus. The arrows indicate sites where the C-terminal part of the protein is interacting with the lipid bilayer.

\section{Involvement of Cytoskeletal Elements, Chromatin and Lipid Biosynthesis in the Formation and Maintenance of Abnormal Nuclear Shapes}

Even though the direct interaction of farnesylated proteins with the lipid components of the NM can nicely explain the effect of lamin Dm0 and Kuk on protein free liposomes, it seems quite unlikely that this is the only event triggering the formation of abnormal nuclear shapes when lamin mutants or farnesylated proteins are accumulating in the nucleus in vivo. First of all, in the case of the liposomes a convex surface is deformed when the farnesylated proteins bind to the lipid layer while in the nucleus they are expected to deform a concave surface, in the case of bleb formation. Furthermore, the complexity of the NM and its interactions with both chromatin and the cytoskeleton suggest that additional cellular components might participate in the induction and maintenance of aberrant nuclear shapes (Fig. 3).

The nucleoskeleton, predominantly consisting of lamins and the cytoskeleton are interconnected via the formation of macromolecular complexes which span the NM. The "linkers of the nucleoskeleton to the cytoskeleton" (LINC) complex primarily consists of SUN proteins of the INM and KASH proteins of the ONM, and mediates the connection of the nuclear lamina to actin filaments, microtubules and centrosomes. ${ }^{40,41}$ Several possibilities of how disturbed nucleoskeleton-cytoskeleton interaction can affect nuclear shape are illustrated in Figure 3A. Mutant forms of lamin accumulating in laminopathic nuclei, might disturb the interactions of the nucleus with cytoskeletal elements, thus affecting nuclear shape. The potential involvement of LINC components in the determination of nuclear shape is highlighted by the abnormal nuclear morphologies observed in the fibroblasts of patients with laminopathies caused by Nesprin mutations, ${ }^{42}$ as well as by the increased levels of SUN1 in HGPS cells ${ }^{43}$ and the decreased levels of Nesprin 2 in a cell culture progeria model. ${ }^{44}$ Actin has also been proposed to define nuclear shape, by forming a cap apical to the nucleus. ${ }^{45}$ This cap is found to be disrupted in cells from laminopathies showing abnormal nuclear shapes as well as in cells with disturbed LINC complexes. Another example where cytoskeletal components have been suggested to be involved in shaping of the nucleus is the neutrophil granulocyte nucleus, in which the centrosome and the associated microtubules are thought to mediate the lobulated nuclear shape. ${ }^{10}$

Conformational changes in chromatin structure might also be partially responsible for shaping the nucleus (Fig. 3B). In our recent study, ${ }^{34}$ we found that downregulation of $\mathrm{BAF}$, an adaptor protein between the lamina and chromatin, ${ }^{46}$ and of p55, a nucleoplasmic WD-repeat component of histone modifying and chromatin assembly complexes, ${ }^{47,48}$ affects nuclear morphology in cultured Drosophila cells. Downregulation of BAF has also been reported to affect nuclear shape in Drosophila imaginal discs and the effect has been attributed to the disruption of interactions between BAF, LEM domain proteins and lamin $\mathrm{Dm} 0 .{ }^{49}$ The interaction of the NM with chromatin is also suggested to participate in the shaping of the granulocyte nucleus, where the elevated levels of LBR are thought to increase the connectivity of NM and chromatin. ${ }^{11}$ Compaction of chromatin together with ER expansion and separation of INM and ONM have been observed upon expression of LBR mutants in human cells in culture. ${ }^{50} \mathrm{NM}$ and chromatin interplay could also explain the abnormal nuclear morphology of Esclp overexpressing yeast cells, since Esclp can function as an anchor for chromatin. ${ }^{36}$

Another mechanism involved in nuclear shape changes could be the expansion of the NM surface by increased incorporation of phospholipids in the bilayer (Fig. 3C). Since phospholipids are one of the main components of membranes, changes involving their biosynthesis, transport or remodeling are expected to affect membrane morphology. As reviewed by Siniossoglou, ${ }^{51}$ lipins, a family of lipid phosphatases involved in the synthesis of diacyl glycerol via dephosphorylation of phosphatidic acid, seem to be involved in nuclear membrane synthesis. Deletion of the yeast lipin PAH1 or of its regulators $N E M 1$ and $S P O 7$, results in the formation of abnormally shaped nuclei with multiple layers of nuclear membrane. ${ }^{52,53}$ Furthermore, downregulation of $C$. elegans lipin, was shown to result in disruption of peripheral ER structure and aberrant nuclear envelope assembly. ${ }^{54}$ 


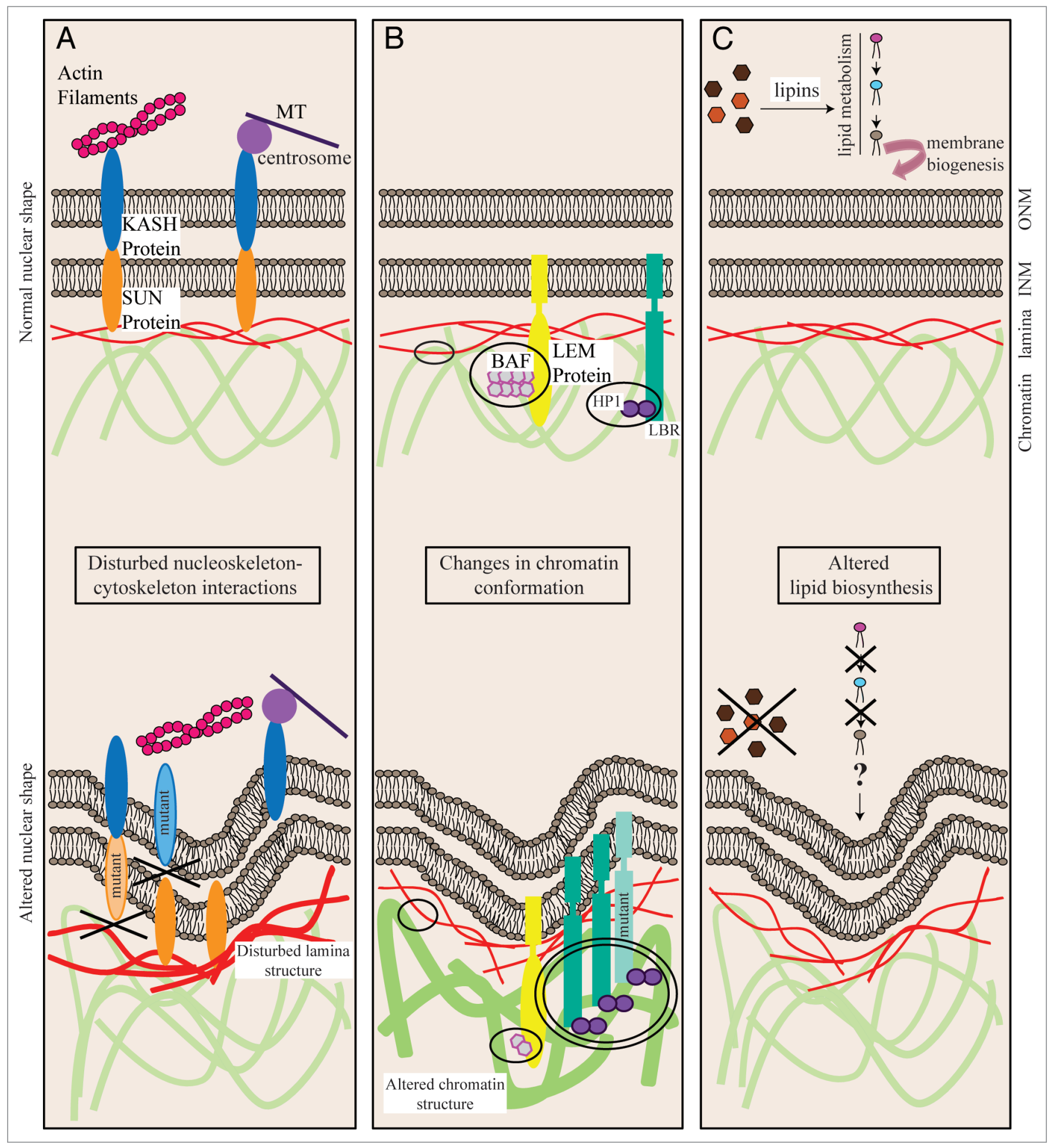

Figure 3. Cytoskeletal elements, chromatin structure and lipid biosynthesis are involved in shaping the nuclear membrane. (A) Disturbed nucleoskeleton-cytoskeleton interactions may be caused i.e., by modified levels of SUN or KASH proteins, by mutations in KASH or SUN proteins or by abnormal lamina structure. In all cases, the interaction of lamina-INM-ONM-cytoskeleton is not properly established. (B) Interactions of the lamina with chromatin (in circles) are either direct or mediated by the interaction of INM proteins with chromatin binding proteins i.e., LEM proteins with BAF ${ }^{46,57}$ or LBR with HP1 ${ }^{58}$ Disturbed interaction of lamina and chromatin either by general changes in chromatin conformation or by modified levels of the proteins mediating the interaction might lead in changes in nuclear shape. The chromatin lamina interaction might be lost (circles) or enhanced i.e., due to increased LBR amounts or to mutations in LBR (double circle). (C) Altered lipid biosynthesis i.e., by mutations in lipins is suggested to influence membrane structure, even though the mechanism remains unclear. 
The influence of lipins in the maintenance of nuclear shape indicates that phospholipid metabolism is connected to nuclear membrane biosynthesis. Even though the exact role of lipins in shaping the nuclear membrane is unclear, it has been suggested that they regulate lipid metabolism at different levels; on one hand by regulating lipid biosynthesis and on the other hand by transcriptionally regulating the expression of genes related to lipid metabolism..$^{51}$ Evidence supporting the latter hypothesis, are the observations that the intranuclear pool of mammalian lipin 1 regulates gene expression of adipogenic and fatty-acid oxidation genes. ${ }^{55,56}$

In conclusion, we propose that a combination of different events including the alteration of NM-chromatin or NM-cytoskeleton interactions and the modification of lipid biosynthesis results in the abnormal nuclear shapes observed upon overexpression of farnesylated nuclear proteins. Even though it is clear, as suggested by experimental data from different systems, that the above mentioned cellular components are involved in shaping the NM, further work is required in order to elucidate the exact molecular mechanism and to analyze the interplay between these different components.

\section{Acknowledgements}

The work was funded by the Deutsche Forschungsgemeinschaft, the Thyssen Foundation and Landesstiftung BadenWürttemberg. M.P. was supported by the Netzwerk Alternsforschung (NAR), University of Heidelberg.

\section{References}

1. Mattout-Drubezki A, Gruenbaum Y. Dynamic interactions of nuclear lamina proteins with chromatin and transcriptional machinery. Cell Mol Life Sci 2003; 60:2053-63.

2. Bridger JM, Foeger N, Kill IR, Herrmann H. The nuclear lamina. Both a structural framework and a platform for genome organization. FEBS J 2007; 274:1354-61.

3. Stewart CL, Roux KJ, Burke B. Blurring the boundary: the nuclear envelope extends its reach. Science 2007; 318:1408-12.

4. Erber A, Riemer D, Bovenschulte M, Weber K. Molecular phylogeny of metazoan intermediate filament proteins. J Mol Evol 1998; 47:751-62.

5. Hattier T, Andrulis ED, Tartakoff AM. Immobility, inheritance and plasticity of shape of the yeast nucleus. BMC Cell Biol 2007; 8:47.

6. Meshorer E, Gruenbaum Y. Gone with the Wnt/ Notch: stem cells in laminopathies, progeria and aging. J Cell Biol 2008; 181:9-13.
7. Chi YH, Chen ZJ, Jeang KT. The nuclear envelopathies and human diseases. J Biomed Sci 2009; 16:96

8. Capell BC, Collins FS. Human laminopathies: nuclei gone genetically awry. Nat Rev 2006; 7:940-52.

9. Mattout A, Dechat T, Adam SA, Goldman RD, Gruenbaum Y. Nuclear lamins, diseases and aging. Curr Opin Cell Biol 2006; 18:335-41.

10. Olins AL, Olins DE. The mechanism of granulocyte nuclear shape determination: possible involvement of the centrosome. Eur J Cell Biol 2005; 84:181-8.

11. Olins AL, Zwerger M, Herrmann $H$, Zentgraf $\mathrm{H}$, Simon AJ, Monestier $\mathrm{M}$, et al. The human granulocyte nucleus: Unusual nuclear envelope and heterochromatin composition. Eur J Cell Biol 2008; 87:279-90

12. Hoffmann K, Sperling K, Olins AL, Olins DE. The granulocyte nucleus and lamin $\mathrm{B}$ receptor: avoiding the ovoid. Chromosoma 2007; 116:227-35.

13. Fabrizio JJ, Hime G, Lemmon SK, Bazinet C. Genetic dissection of sperm individualization in Drosophila melanogaster. Development 1998; 125:1833-43.

14. Abe T, Takano K, Suzuki A, Shimada Y, Inagaki $\mathrm{M}$, Sato N, et al. Myocyte differentiation generates nuclear invaginations traversed by myofibrils associating with sarcomeric protein mRNAs. J Cell Sci 2004; 117:6523-34.

15. Sonnenblick BP. The early embryology of Drosophila melanogaster. In: Biology of Drosophila. Demerec M, ed. Wiley and Son 1950; 62-167.

16. Schejter ED, Wieschaus E. Functional elements of the cytoskeleton in the early Drosophila embryo. Annu Rev Cell Biol 1993; 9:67-99.

17. Lecuit T, Wieschaus E. Polarized insertion of new membrane from a cytoplasmic reservoir during cleavage of the Drosophila embryo. J Cell Biol 2000; 150:849-60.

18. Brandt A, Krohne G, Grosshans J. The farnesylated nuclear proteins KUGELKERN and LAMIN B promote aging-like phenotypes in Drosophila flies. Aging Cell 2008; 7:541-51.

19. Haithcock E, Dayani Y, Neufeld E, Zahand AJ, Feinstein N, Mattout A, et al. Age-related changes of nuclear architecture in Caenorhabditis elegans. Proc Natl Acad Sci USA 2005; 102:16690-5.

20. Scaffidi P, Misteli T. Lamin A-dependent nuclear defects in human aging. Science 2006; 312:1059-63.

21. Zink D, Fischer AH, Nickerson JA. Nuclear structure in cancer cells. Nat Rev Cancer 2004; 4:677-87.

22. Webster M, Witkin KL, Cohen-Fix O. Sizing up the nucleus: nuclear shape, size and nuclear-envelope assembly. J Cell Sci 2009; 122:1477-86.

23. Hoffmann K, Dreger CK, Olins AL, Olins DE, Shultz LD, Lucke B, et al. Mutations in the gene encoding the lamin B receptor produce an altered nuclear morphology in granulocytes (Pelger-Huet anomaly). Nat Genet 2002; 31:410-4.

24. Schirmer EC, Guan T, Gerace L. Involvement of the lamin rod domain in heterotypic lamin interactions important for nuclear organization. J Cell Biol 2001; 153:479-89.

25. Wiesel N, Mattout A, Melcer S, Melamed-Book N, Herrmann H, Medalia O, et al. Laminopathic mutations interfere with the assembly, localization and dynamics of nuclear lamins. Proc Natl Acad Sci USA 2008; 105:180-5.

26. De Sandre-Giovannoli A, Bernard R, Cau P, Navarro C, Amiel J, Boccaccio I, et al. Lamin a truncation in Hutchinson-Gilford progeria. Science 2003; 300:2055.

27. Eriksson M, Brown WT, Gordon LB, Glynn MW, Singer J, Scott L, et al. Recurrent de novo point mutations in lamin A cause Hutchinson-Gilford progeria syndrome. Nature 2003; 423:293-8.

28. Goldman RD, Shumaker DK, Erdos MR, Eriksson M, Goldman AE, Gordon LB, et al. Accumulation of mutant lamin A causes progressive changes in nuclear architecture in Hutchinson-Gilford progeria syndrome. Proc Natl Acad Sci USA 2004; 101:8963-8.
29. Yang SH, Bergo MO, Toth JI, Qiao X, Hu Y, Sandoval $\mathrm{S}$, et al. Blocking protein farnesyltransferase improves nuclear blebbing in mouse fibroblasts with a targeted Hutchinson-Gilford progeria syndrome mutation. Proc Natl Acad Sci USA 2005; 102:10291-6.

30. Toth JI, Yang SH, Qiao X, Beigneux AP, Gelb MH, Moulson CL, et al. Blocking protein farnesyltransferase improves nuclear shape in fibroblasts from humans with progeroid syndromes. Proc Natl Acad Sci USA 2005; 102:12873-8.

31. Ralle T, Grund C, Franke WW, Stick R. Intranuclear membrane structure formations by $\mathrm{CaaX}$-containing nuclear proteins. J Cell Sci 2004; 117:6095-104.

32. Prufert K, Vogel A, Krohne G. The lamin CxxM motif promotes nuclear membrane growth. J Cell Sci 2004; 117:6105-16.

33. Brandt A, Papagiannouli F, Wagner N, WilschBrauninger $M$, Braun $M$, Furlong EE, et al. Developmental control of nuclear size and shape by Kugelkern and Kurzkern. Curr Biol 2006; 16:543-52.

34. Polychronidou M, Hellwig A, Grosshans J. The farnesylated nuclear proteins kugelkern and lamin $\mathrm{Dm} 0$ affect nuclear morphology by directly interacting with the nuclear membrane. Mol Biol Cell 2010; 21:3409-20.

35. Pilot F, Philippe JM, Lemmers C, Chauvin JP, Lecuit T. Developmental control of nuclear morphogenesis and anchoring by charleston, identified in a functional genomic screen of Drosophila cellularisation. Development 2006; 133:711-23.

36. Taddei A, Hediger F, Neumann FR, Bauer C, Gasser SM. Separation of silencing from perinuclear anchoring functions in yeast Ku80, Sir4 and Escl proteins. EMBO J 2004; 23:1301-12.

37. Firmbach-Kraft I, Stick R. The role of CaaXdependent modifications in membrane association of Xenopus nuclear lamin B3 during meiosis and the fate of B3 in transfected mitotic cells. J Cell Biol 1993; 123:1661-70.

38. Nigg EA, Kitten GT, Vorburger K. Targeting lamin proteins to the nuclear envelope: the role of CaaX box modifications. Biochem Soc Trans 1992; 20:500-4.

39. Hennekes H, Nigg EA. The role of isoprenylation in membrane attachment of nuclear lamins. A single point mutation prevents proteolytic cleavage of the lamin A precursor and confers membrane binding properties. J Cell Sci 1994; 107:1019-29.

40. Razafsky D, Hodzic D. Bringing KASH under the SUN: the many faces of nucleo-cytoskeletal connections. J Cell Biol 2009; 186:461-72.

41. Fridkin A, Penkner A, Jantsch V, Gruenbaum Y. SUN-domain and $\mathrm{KASH}$-domain proteins during development, meiosis and disease. Cell Mol Life Sci 2009; 66:1518-33.

42. Zhang Q, Bethmann C, Worth NF, Davies JD, Wasner C, Feuer A, et al. Nesprin-1 and -2 are involved in the pathogenesis of Emery Dreifuss muscular dystrophy and are critical for nuclear envelope integrity. Hum Mol Genet 2007; 16:2816-33.

43. Haque F, Mazzeo D, Patel JT, Smallwood DT, Ellis JA, Shanahan CM, et al. Mammalian SUN protein interaction networks at the inner nuclear membrane and their role in laminopathy disease processes. J Biol Chem 2010; 285:3487-98.

44. Kandert S, Luke Y, Kleinhenz T, Neumann S, Lu W, Jaeger VM, et al. Nesprin-2 giant safeguards nuclear envelope architecture in LMNA S143F progeria cells. Hum Mol Genet 2007; 16:2944-59.

45. Khatau SB, Hale CM, Stewart-Hutchinson PJ, Patel MS, Stewart CL, Searson PC, et al. A perinuclear actin cap regulates nuclear shape. Proc Natl Acad Sci USA 2009; 106:19017-22.

46. Margalit A, Brachner A, Gotzmann J, Foisner R, Gruenbaum Y. Barrier-to-autointegration factora BAFfling little protein. Trends Cell Biol 2007; 17:202-8. 
47. Tyler JK, Bulger M, Kamakaka RT, Kobayashi R, Kadonaga JT. The p55 subunit of Drosophila chromatin assembly factor 1 is homologous to a histone deacetylase-associated protein. Mol Cell Biol 1996; 16:6149-59.

48. Tyler JK, Collins KA, Prasad-Sinha J, Amiott E, Bulger M, Harte PJ, et al. Interaction between the Drosophila CAF-1 and ASF1 chromatin assembly factors. Mol Cell Biol 2001; 21:6574-84.

49. Furukawa K, Sugiyama S, Osouda S, Goto H, Inagaki $\mathrm{M}$, Horigome T, et al. Barrier-to-autointegration factor plays crucial roles in cell cycle progression and nuclear organization in Drosophila. J Cell Sci 2003; 116:3811-23.

50. Zwerger M, Kolb T, Richter K, Karakesisoglou I, Herrmann H. Induction of a massive endoplasmic reticulum and perinuclear space expansion by expression of lamin B receptor mutants and the related sterol reductases TM7SF2 and DHCR7. Mol Biol Cell 2010; 21:354-68.

51. Siniossoglou S. Lipins, lipids and nuclear envelope structure. Traffic 2009; 10:1181-7.
52. Santos-Rosa H, Leung J, Grimsey N, Peak-Chew S, Siniossoglou S. The yeast lipin Smp2 couples phospholipid biosynthesis to nuclear membrane growth. EMBO J 2005; 24:1931-41.

53. Siniossoglou S, Santos-Rosa H, Rappsilber J, Mann M, Hurt E. A novel complex of membrane proteins required for formation of a spherical nucleus. EMBO J 1998; 17:6449-64.

54. Golden A, Liu J, Cohen-Fix O. Inactivation of the C. elegans lipin homolog leads to ER disorganization and to defects in the breakdown and reassembly of the nuclear envelope. J Cell Sci 2009; 122:1970-8.

55. Finck BN, Gropler MC, Chen Z, Leone TC, Croce MA, Harris TE, et al. Lipin 1 is an inducible amplifier of the hepatic PGC-1alpha/PPARalpha regulatory pathway. Cell Metab 2006; 4:199-210.
56. Koh YK, Lee MY, Kim JW, Kim M, Moon JS, Lee YJ, et al. Lipin1 is a key factor for the maturation and maintenance of adipocytes in the regulatory network with CCAAT/enhancer-binding protein alpha and peroxisome proliferator-activated receptor gamma2. J Biol Chem 2008; 283:34896-906.

57. Segura-Totten M, Wilson KL. BAF: roles in chromatin, nuclear structure and retrovirus integration. Trends Cell Biol 2004; 14:261-6.

58. Ye Q, Callebaut I, Pezhman A, Courvalin JC, Worman HJ. Domain-specific interactions of human HP1-type chromodomain proteins and inner nuclear membrane protein LBR. J Biol Chem 1997; 272:14983-9. 